\title{
Average Conditions for the Permanence of a Bounded Discrete Predator-Prey System
}

\author{
Yong-Hong Fan and Lin-Lin Wang \\ School of Mathematics and Statistics Science, Ludong University, Yantai, Shandong 264025, China \\ Correspondence should be addressed to Yong-Hong Fan; fanyh_1993@sina.com
}

Received 21 June 2013; Accepted 18 July 2013

Academic Editor: Antonia Vecchio

Copyright (C) 2013 Y.-H. Fan and L.-L. Wang. This is an open access article distributed under the Creative Commons Attribution License, which permits unrestricted use, distribution, and reproduction in any medium, provided the original work is properly cited.

Average conditions are obtained for the permanence of a discrete bounded system with Holling type II functional response $u(n+1)=$ $u(n) \exp \{a(n)-b(n) u(n)-c(n) v(n) /(u(n)+m(n) v(n))\}, v(n+1)=v(n) \exp \{-d(n)+e(n) u(n) /(u(n)+m(n) v(n))\}$. The method involves the application of estimates of uniform upper and lower bounds of solutions. When these results are applied to some special delay population models with multiple delays, some new results are obtained and some known results are generalized.

\section{Introduction}

In this paper, we will study the permanence of the following discrete system:

$$
\begin{aligned}
& u(n+1) \\
& =u(n) \exp \left\{a(n)-b(n) u(n)-\frac{c(n) v(n)}{u(n)+m(n) v(n)}\right\}, \\
& v(n+1)=v(n) \exp \left\{-d(n)+\frac{e(n) u(n)}{u(n)+m(n) v(n)}\right\},
\end{aligned}
$$

where the sequences $a(n), b(n), c(n), d(n), e(n), m(n)$ are all assumed to be bounded and $b(n), c(n), e(n), m(n)$ are all positive for $n \in \mathbb{Z}$.

If all the coefficients of the previous system (1) are periodic sequences with period $\omega$, in [1], the authors obtained the following.

Theorem 1 (see [1]). Assume that

$$
\bar{a}>\overline{\left(\frac{c}{m}\right)}, \quad \bar{e}>\bar{d},
$$

hold; then the periodic system (1) is permanent.

In [2], by a standard comparison argument, they proved the following.
Theorem 2. Assume that

$$
a^{L}>\frac{c^{M}}{m^{L}}, \quad e^{L}>d^{M},
$$

hold; then the bounded system (1) is permanent.

In the previous two theorems, we used the denotation as follows. For a bounded sequence $g(n)$, we define

$$
\begin{aligned}
g^{M} & =\sup \{g(k) \mid k \in \mathbb{Z}\}, \\
g^{L} & =\inf \{g(k) \mid k \in \mathbb{Z}\} .
\end{aligned}
$$

And for a given periodic sequence with period $\omega$, its average value is defined as

$$
\bar{f}=\frac{1}{\omega} \sum_{i=0}^{\omega-1} f(i)
$$

Throughout this paper, we always assume that $b^{L}>$ $0, c^{L}>0, e^{L}>0, m^{L}>0$.

If all the coefficients of system (1) are periodic sequences with period $\omega$, then it is a special form of the bounded coefficients of system (1), but from Theorem 2, we cannot obtain Theorem 1; that is to say, there is a gap between Theorems 1 and 2. In this paper, we attempt to fill in this gap. 
In order to illustrate our main results, similar to the corresponding definitions of the bounded continuous function in [3], we first introduce some notations.

For a bounded sequence $f: \mathbb{Z} \rightarrow \mathbb{R}$, we define the lower average of $f$ by

$$
A_{L}(f)=\lim _{r \rightarrow \infty} \inf _{t-s \geq r} \frac{1}{t-s} \sum_{k=s}^{t} f(k) .
$$

Some remarks:

(a) For a bounded sequence $f$, define the upper average $A_{M}(f)$ of $f$ by replacing inf with sup in (6).

(b) If $f$ is $\omega$-periodic, then

$$
A_{L}(f)=A_{M}(f)=\bar{f} .
$$

(c) The following inequalities hold true:

$$
f^{L} \leq A_{L}(f) \leq A_{M}(f) \leq f^{M} .
$$

(d) For any $\alpha, \beta \in R$, the lower average satisfies

$$
A_{L}(\alpha f+\beta g)=\alpha A_{L}(f)+\beta A_{L}(g) .
$$

Proof. We only prove that (b) hold; (c) and (d) can be proved similarly as that in [3]. Setting $t-s=n \omega+\alpha_{n}$, where $\alpha_{n} \epsilon$ $[0, \omega-1]$, in the following, we assume that $n$ is sufficiently large; then

$$
\begin{aligned}
\frac{1}{t-s} \sum_{k=s}^{t} f(k) & =\frac{1}{n \omega+\alpha_{n}} \sum_{k=s}^{s+n \omega-1} f(k)+\frac{1}{n \omega+\alpha_{n}} \sum_{k=s+n \omega}^{t} f(k) \\
& =\frac{n}{n \omega+\alpha_{n}} \sum_{k=0}^{\omega-1} f(k)+\frac{\left(\alpha_{n}+1\right) f^{M}}{n \omega+\alpha_{n}},
\end{aligned}
$$

from the previous equality, we have

$$
\lim _{n \rightarrow \infty} \frac{1}{t-s} \sum_{k=s}^{t} f(k)=\frac{1}{\omega} \sum_{k=0}^{\omega-1} f(k)
$$

therefore

$$
A_{L}(f)=A_{M}(f)=\frac{1}{\omega} \sum_{k=0}^{\omega-1} f(k)=\bar{f}
$$

which completes the proof.

During the study of the permanence for the bounded system, in view of the property (b), one can usually use the lower average or upper average instead of the sup and inf values. And we call the condition obtained by using the method of lower average or upper average as "average conditions." For the permanence results with "average conditions," one can refer to [4-7], and so forth.

For the permanence of system (1), we have the following.
Theorem 3. Assume that

$$
\begin{array}{r}
A_{L}\left(a(n)-\frac{c(n)}{m(n)}\right)>0, \quad A_{L}(e(n)-d(n))>0, \\
A_{L}(d)>0 ;
\end{array}
$$

then the bounded system (1) is permanent.

Obviously, Theorem 3 includes both Theorems 1 and 2 . Therefore, this theorem is a bridge that combines the bounded system and the periodic system.

\section{Preliminaries}

In order to prove Theorem 3, we need some lemmas below. The first lemma could be found in [8].

Lemma 4 (see [8, Corollary 2.5]). Let $u(n)$ be a positive solution of the following inequality:

$$
\begin{gathered}
u(n+1) \leq u(n) \exp \left\{a_{1}(n)-b_{1}(n) u(n)\right\}, \quad n \in \mathbb{Z}, \\
u(0)>0,
\end{gathered}
$$

if $b_{1}^{L}>0$ and $a_{1}^{M}>0$; then

$$
\lim \sup _{n \rightarrow \infty} u(n) \leq \min \left\{\frac{a_{1}^{M}}{b_{1}^{L}} \exp \left\{a_{1}^{M}\right\}, \frac{1}{b_{1}^{L}} \exp \left\{a_{1}^{M}-1\right\}\right\} .
$$

We should point out that when $b_{1}^{L}=0$, the conclusion of the previous lemma is not true. That is, $b_{1}^{L}>0$ is a necessary condition. We give an example to illustrate it.

Example 5. Consider the following inequality:

$$
\begin{gathered}
u(n+1) \leq u(n) \exp \left\{\frac{1}{2}+\frac{1}{n}-\frac{1}{n} u(n)\right\}, \quad n \in \mathbb{Z}, \\
u(0)>0 .
\end{gathered}
$$

Obviously, $u(n)=n$ is a solution of it, but $\lim \sup _{n \rightarrow \infty} u(n)=$ $+\infty$.

Lemma 6. Let $u(n)$ be a solution of the following inequality:

$$
\begin{gathered}
u(n+1) \geq u(n) \exp \left\{a_{2}(n)-b_{2}(n) u(n)\right\}, \quad n \in \mathbb{Z}, \\
u(0)>0,
\end{gathered}
$$

and bounded above; if $b_{2}^{L}>0$ and

$$
A_{L}\left(a_{2}\right)>0
$$

then there exists some positive constant L such that

$$
\lim \inf _{n \rightarrow \infty} u(n) \geq L
$$

To prove this lemma, we give two claims in what follows. First, by using mathematical induction, we can easily obtain the following. 
Claim 1. If $u(n)$ is a solution of (17), then

$$
u(n)>0 \quad \text { for any } n \in \mathbb{Z} \text {. }
$$

In what follows, we use contradiction to prove the lemma.

Claim 2. Assume that $u(k)$ is a solution of (17) and bounded above by a positive constant $M$; if (19) does not hold, then there exist positive integer sequences $\left\{s_{n}\right\}$ and $\left\{t_{n}\right\}$ such that

$$
\begin{gathered}
0 \leq s_{n}<t_{n}, \quad t_{n}-s_{n} \geq n+1, \quad u\left(s_{n}\right) \geq \frac{u(0)}{n}, \\
u(k) \leq \frac{u(0)}{n} \text { for } s_{n}<k \leq t_{n} .
\end{gathered}
$$

Proof of the claim. Notice that

$$
a_{2}(n)-b_{2}(n) u(n) \geq a_{2}^{L}-b_{2}^{M} M \geq-\gamma \quad \text { forany } n \in \mathbb{Z} \text {, }
$$

where $\gamma>0$ is a constant.

If (19) does not hold, then from Claim 1, $\lim \inf _{n \rightarrow \infty} u(n)=0$, thus, for any positive integer $n \geq 1$, there exist $t_{n}>0$ such that

$$
u\left(t_{n}\right)<\frac{u(0)}{n} \exp \{-n \gamma\}
$$

In addition, there exists a number $s_{n}$ such that $0 \leq s_{n}<t_{n}$, $u\left(s_{n}\right) \geq u(0) / n$ and $u(k) \leq u(0) / n$ for $s_{n}<k \leq t_{n}$. In the following, we only need to prove that $t_{n}-s_{n} \geq n+1$. From the first equation of (17), we have

$$
\begin{aligned}
\frac{u(0)}{n} & \leq u\left(s_{n}\right) \leq u\left(t_{n}\right) \exp \left\{-\sum_{k=s_{n}}^{t_{n}-1}\left(a_{2}(k)-b_{2}(k) u(k)\right)\right\} \\
& \leq u\left(t_{n}\right) \exp \left\{\gamma\left(t_{n}-s_{n}-1\right)\right\} \\
& \leq \frac{u(0)}{n} \exp \{-n \gamma\} \exp \left\{\gamma\left(t_{n}-s_{n}-1\right)\right\},
\end{aligned}
$$

which implies that $t_{n}-s_{n} \geq n+1$. This completes the proof of Claim 2.

Proof of Lemma 6. From the first equation of (17), we have

$$
a_{2}(k) \leq \ln \frac{u(k+1)}{u(k)}+b_{2}(k) u(k),
$$

by Claim 2, we obtain that if (19) does not hold, then for any $n \geq 1$, we have

$$
\sum_{k=s_{n}}^{t_{n}-1} a_{2}(k) \leq \sum_{k=s_{n}}^{t_{n}-1} \ln \frac{u(k+1)}{u(k)}+\sum_{k=s_{n}}^{t_{n}-1} b_{2}(k) u(k),
$$

which implies that

$$
\begin{aligned}
& \frac{1}{t_{n}-s_{n}-1} \sum_{k=s_{n}}^{t_{n}-1} a_{2}(k) \\
& \leq \frac{1}{t_{n}-s_{n}-1} \ln \frac{u\left(t_{n}\right)}{u\left(s_{n}\right)}+\frac{b_{2}\left(s_{n}\right) u\left(s_{n}\right)}{t_{n}-s_{n}-1}+\frac{t_{n}-s_{n}-2}{t_{n}-s_{n}-1} \frac{u(0) b_{2}^{M}}{n} \\
& <\frac{-n \gamma}{t_{n}-s_{n}-1}+\frac{b_{2}\left(s_{n}\right) u\left(s_{n}\right)}{t_{n}-s_{n}-1}+\frac{t_{n}-s_{n}-2}{t_{n}-s_{n}-1} \frac{u(0) b_{2}^{M}}{n} .
\end{aligned}
$$

Notice that

$$
\begin{gathered}
\lim _{n \rightarrow \infty} \frac{b_{2}\left(s_{n}\right) u\left(s_{n}\right)}{t_{n}-s_{n}-1}=0, \\
\lim _{n \rightarrow \infty} \frac{t_{n}-s_{n}-2}{t_{n}-s_{n}-1} \frac{u(0) b_{2}^{M}}{n}=0 ;
\end{gathered}
$$

thus, by (27), we have

$$
\lim _{n \rightarrow \infty} \frac{1}{t_{n}-s_{n}-1} \sum_{k=s_{n}}^{t_{n}-1} a_{2}(k) \leq 0 .
$$

This is in contradiction to (18); the proof is complete.

From Lemmas 4 and 6, we have the following.

Theorem 7. Let $u(n)$ be a solution of the following inequality:

$$
\begin{aligned}
& u(n) \exp \left\{a_{2}(n)-b_{2}(n) u(n)\right\} \\
& \leq u(n+1) \leq u(n) \exp \left\{a_{1}(n)-b_{1}(n) u(n)\right\}, \\
& n \in \mathbb{Z}, \quad u(0)>0 ;
\end{aligned}
$$

if

$$
b_{1}^{L}>0, \quad b_{2}^{L}>0, \quad A_{L}\left(a_{2}\right)>0,
$$

then there exist some positive constants $L$ and $M$ such that

$$
L \leq \lim \inf _{t \rightarrow \infty} u(t) \leq \lim \sup _{t \rightarrow \infty} u(t) \leq M .
$$

From Theorem 7, we can easily obtain the following.

Corollary 8. Let $u(n)$ be a solution of the following inequality:

$$
\begin{aligned}
& u(n) \exp \left\{a_{2}(n)-b_{2}(n) u(n-k)\right\} \\
& \quad \leq u(n+1) \leq u(n) \exp \left\{a_{1}(n)-b_{1}(n) u(n-k)\right\},
\end{aligned}
$$

for any $n \in \mathbb{Z}, u(i)>0,-k+1 \leq i \leq 0$. If

$$
b_{1}^{L}>0, \quad b_{2}^{L}>0, \quad A_{L}\left(a_{2}\right)>0
$$

then the conclusion of Theorem 7 also holds true, where $k$ is a positive integer. 


\section{Permanence}

In this section, we give some applications of Theorem 7. First we use it to prove Theorem 3.

Proof of Theorem 3. From the first equation of (1), we have

$$
\begin{aligned}
& u(n) \exp \left\{a(n)-\frac{c(n)}{m(n)}-b(n) u(n)\right\} \\
& \quad \leq u(n+1) \leq u(n) \exp \{a(n)-b(n) u(n)\} ;
\end{aligned}
$$

by Theorem 7 and the condition (13), we can obtain that there must exist some positive constants $L_{1}$ and $M_{1}$ such that

$$
L_{1} \leq \lim \inf _{n \rightarrow \infty} u(n) \leq \lim \sup _{n \rightarrow \infty} u(n) \leq M_{1}
$$

for any solution $(u(n), v(n))$ of (1) with positive initial conditions $u(0)>0$ and $v(0)>0$.

From the second equation of (1), we have

$$
\begin{aligned}
v(n+1) & =v(n) \exp \left\{e(n)-d(n)-\frac{e(n) m(n) v(n)}{u(n)+m(n) v(n)}\right\} \\
& \geq v(t)\left[e(n)-d(n)-\frac{e(n) m(n) v(n)}{L_{1}}\right]
\end{aligned}
$$

by Theorem 7 and condition (13), we can obtain that there exists a positive constant $L_{2}$ such that

$$
\lim \inf _{n \rightarrow \infty} v(n) \geq L_{2} .
$$

Set $y(n)=1 / v(n)$; then from the second equation of (1), we can obtain

$$
\begin{aligned}
y(n+1) & =y(n) \exp \left\{d(n)-\frac{e(n) u(n)}{u(n) y(n)+m(n)} y(n)\right\} \\
& \geq y(n) \exp \left\{d(n)-\frac{e(n) M_{1}}{m(n)} y(n)\right\},
\end{aligned}
$$

for sufficiently large $t$; by Theorem 7 and (13), we have

$$
\lim \sup _{n \rightarrow \infty} v(n) \leq M_{2} .
$$

By (36), (38), and (40), we complete the proof.

Through some similar analysis as in [9], we have the following.

Corollary 9. Assume that any positive solution of the periodic equation $u(n+1)=f(n, u(n))(f(n+\omega, u)=f(n, \omega), \forall n \in \mathbb{Z})$ satisfies

$$
\begin{aligned}
& u(n) \exp \left\{a_{2}(n)-b_{2}(n) u(n)\right\} \\
& \leq u(n+1) \leq u(n) \exp \left\{a_{1}(n)-b_{1}(n) u(n)\right\}, \\
& n \in \mathbb{Z}, \quad u(0)>0,
\end{aligned}
$$

where $a_{i}(n), b_{i}(n)(i=1,2)$ are all $\omega$-periodic sequences; if

$$
b_{1}^{L}>0, \quad b_{2}^{L}>0, \quad \overline{a_{2}}>0,
$$

then the periodic equation $u(n+1)=f(n, u(n))$ has at least one $\omega$-periodic positive solution.

We should point out that the previous corollary can be generalized to the $n$-dimensional situation. As a direct application of the previous corollary, we have the following.

Theorem 10. Assume that

$$
\bar{a}>\overline{\left(\frac{c}{m}\right)}, \quad \bar{e}>\bar{d}>0,
$$

hold; then the periodic system (1) (the coefficients of the system (1) are all periodic sequences with a common period $\omega$ ) has at least one $\omega$-periodic positive solution.

This theorem generalized Theorem 3.1 in [10].

\section{Acknowledgments}

This work was supported by the Natural Science Foundation of China (11201213), the Natural Science Foundation of Shandong Province (ZR2010AM022), and the Outstanding Young and Middle-Aged Scientists Research Award Fund of Shandong Province (BS2011SF004).

\section{References}

[1] Y. Fan and W. Li, "Harmless delays in a discrete ratio-dependent periodic predator-prey system," Discrete Dynamics in Nature and Society, vol. 2006, Article ID 12176, 21 pages, 2006.

[2] H. Huo and W. Li, "Permanence and global stability of positive solutions of a nonautonomous discrete ratio-dependent predator-prey model," Discrete Dynamics in Nature and Society, vol. 2005, no. 2, pp. 135-144, 2005.

[3] A. Tineo, "An iterative scheme for the $\mathrm{N}$-competing species problem," Journal of Differential Equations, vol. 116, no. 1, pp. $1-15,1995$.

[4] S. Ahmad and A. Lazer, "Average growth and extinction in a competitive Lotka-Volterra system," Nonlinear Analysis, Theory, Methods and Applications, vol. 62, no. 3, pp. 545-557, 2005.

[5] F. Chen, "Average conditions for permanence and extinction in nonautonomous Gilpin-Ayala competition model," Nonlinear Analysis, Real World Applications, vol. 7, no. 4, pp. 895-915, 2006.

[6] J. Zhao and J. Jiang, "Average conditions for permanence and extinction in nonautonomous Lotka-Volterra system," Journal of Mathematical Analysis and Applications, vol. 299, no. 2, pp. 663-675, 2004.

[7] S. Ahmad and A. Lazer, "Average conditions for global asymptotic stability in a nonautonomous Lotka-Volterra system," Nonlinear Analysis, Theory, Methods and Applications, vol. 40, no. 1, pp. 37-49, 2000.

[8] Y. Fan and L. Wang, "On a generalized discrete ratio-dependent prey-predator system," Discrete Dynamics in Nature and Society, vol. 2009, Article ID 653289, 22 pages, 2009. 
[9] Z. Teng and L. Chen, "The positive solutions in periodic Kolmogorov type systems with delay," Acta Mathematicae Applicatae Sinica, vol. 22, pp. 446-456, 1999 (Chinese).

[10] M. Fan and K. Wang, "Periodic solutions of a discrete time nonautonomous ratio-dependent predator-prey system," Mathematical and Computer Modelling, vol. 35, no. 9-10, pp. 951-961, 2002. 


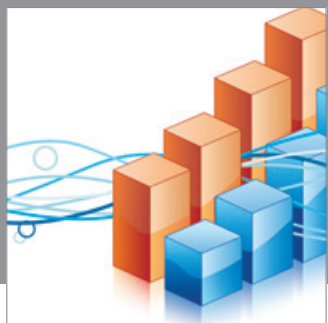

Advances in

Operations Research

mansans

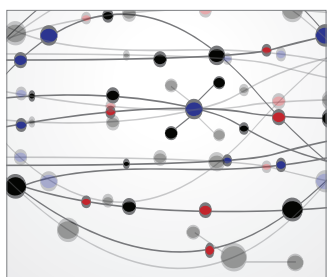

The Scientific World Journal
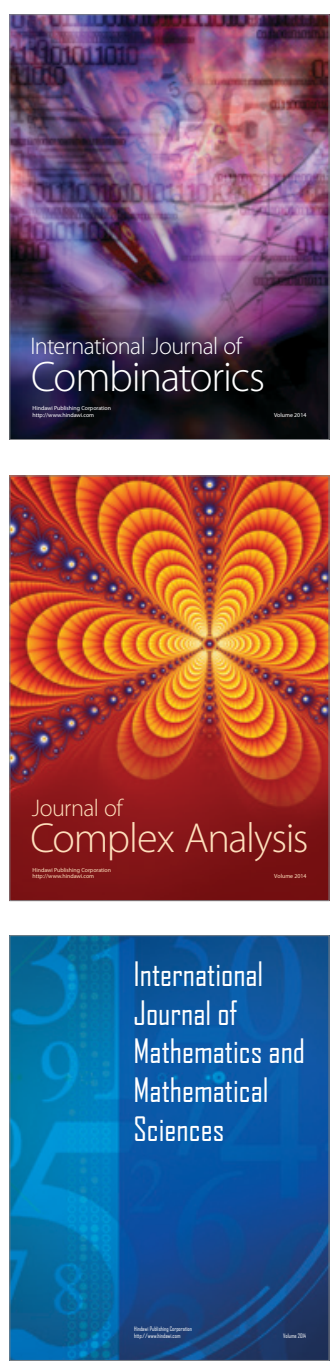
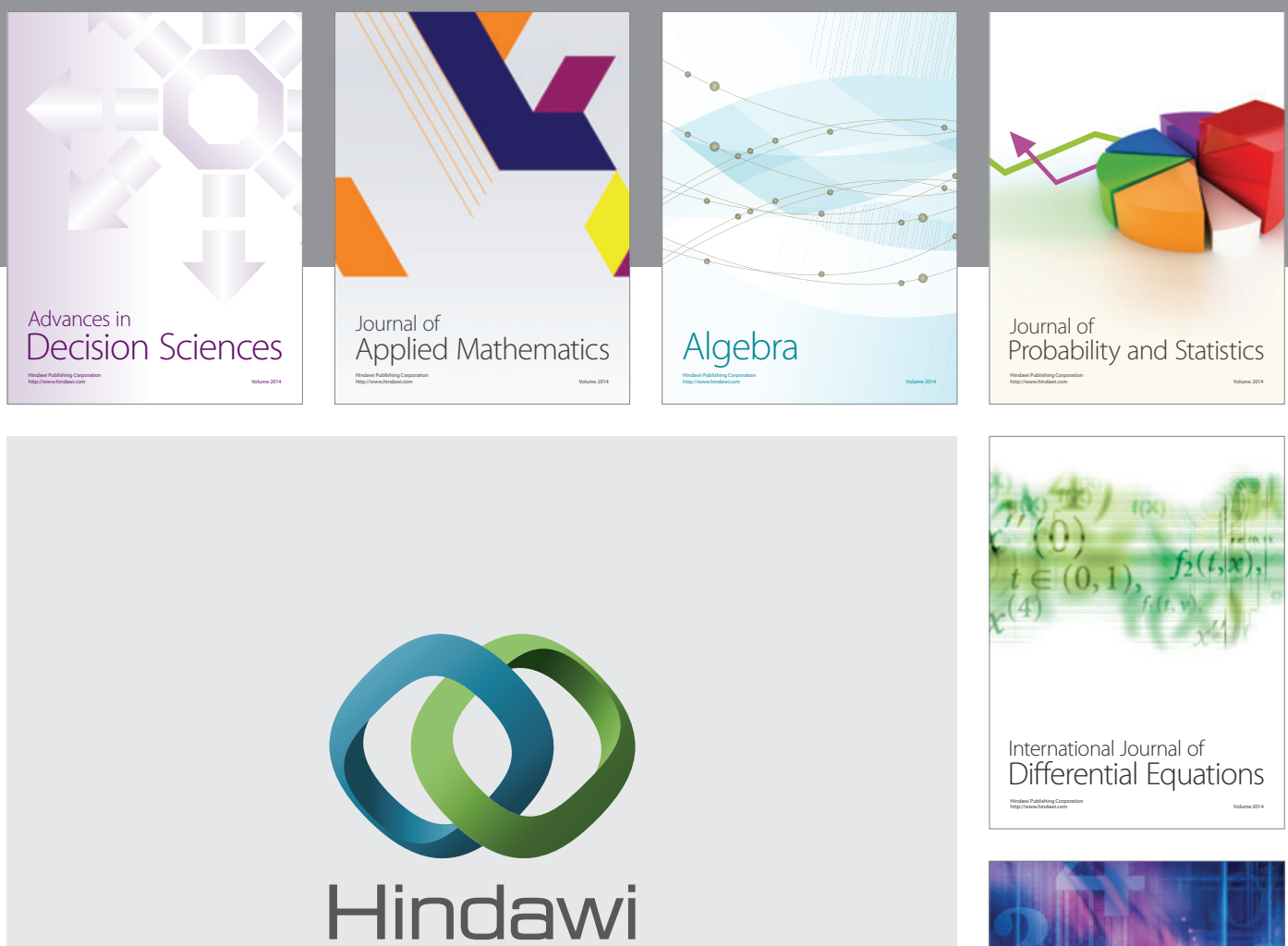

Submit your manuscripts at http://www.hindawi.com
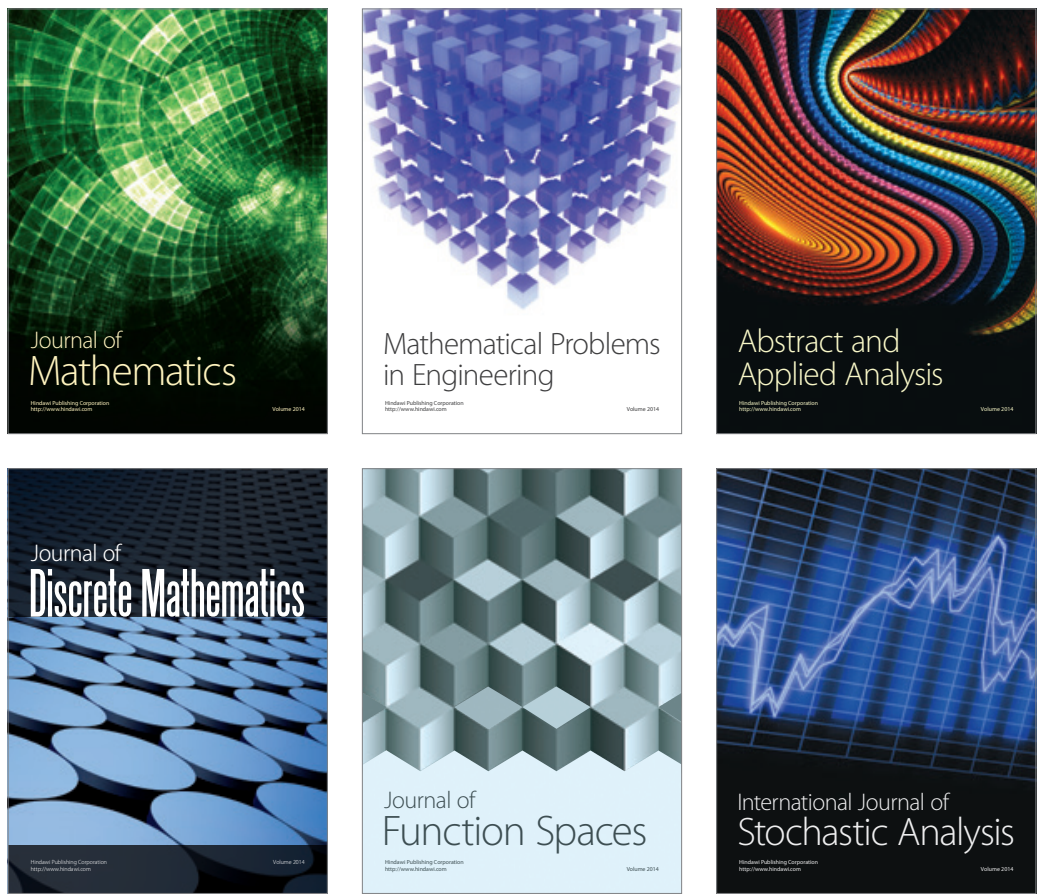

Journal of

Function Spaces

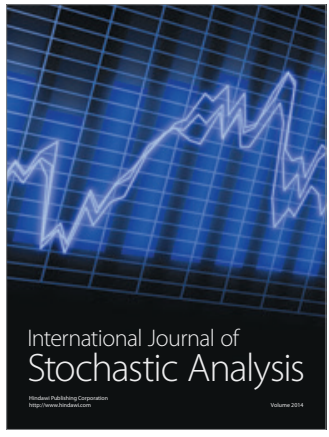

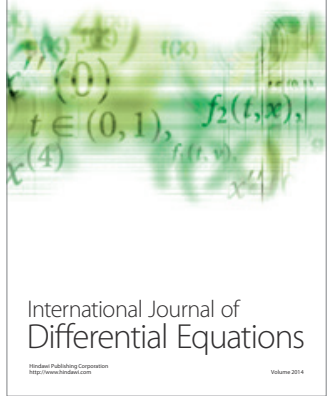
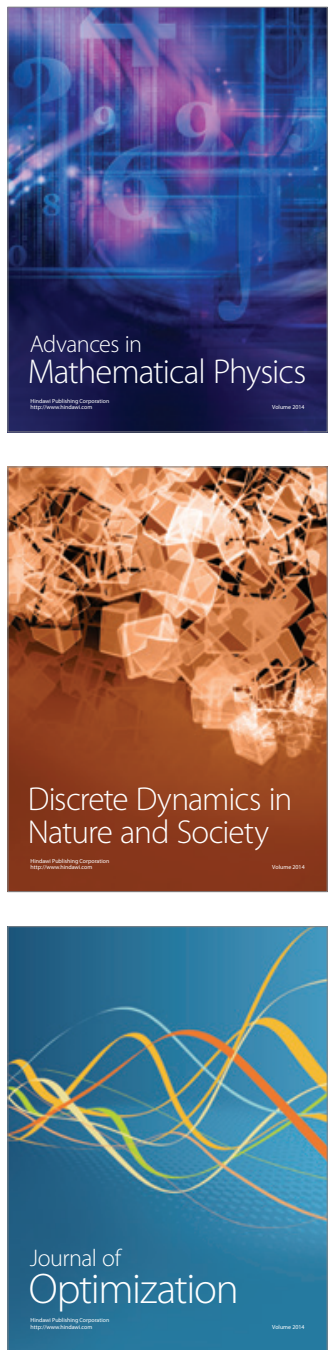